\title{
Surgical excision of schwannoma arising from the infraorbital nerve trunk via vestibular approach (a
} case report)

\begin{abstract}
Schwannoma is a benign neural neoplasm of Schwann cell origin. It is relatively uncommon, although $25 \%$ to $48 \%$ of all cases occur in the head and neck region. However, schwannoma originating from infraorbital nerve is rarely seen and reported. The goal of this study is to report a rare case of schwannoma that arises from the infraorbital nerve trunk that surgically excised via vestibular approach. A 41 y.o male patient came with a mass at the right buccal region since about six months before admission. The lump getting bigger slowly from a marble size into a pingpong ball size. Clinical examination reveals a mass at the buccal region with firm consistency that can also be palpated at the right vestibular region. Incision biopsy was performed and histopathological report was schwannoma. Subsequently surgical excision via vestibular approach was performed to remove the mass. At the time of exploration, the mass was observed to emanate from the terminal of the infraorbital nerve trunk. Schwannoma arising from the infraorbital nerve trunk is a rare case in the oral and maxillofacial region. This tumor usually present as a slow growing nodular mass. Schwannoma arising from terminal trunk of infraorbital nerve is best surgically removed via vestibular approach to eliminate the post operation scar.
\end{abstract}

Volume 9 Issue 3 - 2018

\section{Irsan Kurniawan, Mantra Nandini,Andri Hardianto}

Department of Oral and Maxillofacial Surgery, University of Padjadjaran, Indonesia

Correspondence: Irsan Kurniawan, Faculty of Dentistry, Department of Oral and Maxillofacial Surgery, University of Padjadjaran, Indonesia, Email irsankurniawan82@gmail.com

Received: July 26, 2017 | Published: May 14, 2018

Keywords: schwannoma, infraorbital nerve, surgical excision

\section{Introduction}

Schwannoma is a benign neural neoplasm of schwann cell origin that is an axon sheath cells. Generally the tumor is well demarcated, consisting of a fibroblastic proliferation of the nerve sheath cell. ${ }^{1-4}$ Although $25 \%$ to $48 \%$ of all schwannoma cases occur in the head and neck region, ${ }^{2}$ however it is rarely involves infra-orbital nerve and are uncommon in the maxillary and buccal space accounting for less than $4 \%$. Schwannomas can occur at any age but are most common between 30 and 50 years. ${ }^{1}$ There is no specific sex predilection for this pathologic lesion discussed in several literatures. There is no clear aetiology of a schwannoma, the pathogenesis is not discussed in oral and maxillofacial pathology literatures. Two types of schwannomas have been identified, first peripheral which is located in the soft tissues and second, central or intraosseous which is located within the bone ${ }^{1-4}$ They rarely will occur centrally within the mandible or maxilla and give rise to a unilocular expansile radiolucency. Soft tissue tumors will present as discrete, freely movable masses, firm and seem to have a smooth surface. ${ }^{1,4}$ A CT or MRI scan readily implies a schwannoma if it shows an oval, mostly homogenous, soft tissue mass with a pericapsular fat plane. ${ }^{1}$ Histopathologically, the appearance is distinctive with multiple rounded masses of elongated spindle cells with palisaded nuclei (Antoni A tissue). There is also a variable amount of unorganised loose connective tissue with scanty cells, sometimes with pleomorphic nuclei (Antoni B tissue). ${ }^{1-4}$ Surgical excision is the treatment of choice for schwannoma. Because tumors are well demarcated and encapsulated, they are easily dissected from the surrounding tissue. Some authors believe that no recurrence may occur after complete removal of the mass. ${ }^{1,2}$ But some authors put the recurrence rates are less than $5 \%$ until $10 \% .{ }^{1,4}$ This paper describes a case of schwannoma arising from infraorbital nerve, presenting as slow growing nodular swelling in the buccal space. The surgical excision was performed via intraoral vestibular approach and we did exploration through the mass. At the time of exploration, the lesion was observed to emanate from the infra-orbital nerve trunk.

\section{Case report}

A 41 y.o male patient came with a mass at the right buccal region. The patient felt that mass arises about six months before admission. The lump getting bigger slowly from a marble size to a pingpong ball size. There was no other lump noted in other parts of the patients body. There was no pain experienced by the patient, instead of numbness in the area of right cheek and upper lip region. There was no nasal obstruction complain by the patient, and the eyes was also within normal limit. Extraoral examination we found facial asymmetry because of there was a mass at the right buccal region with normal skin surface above the mass. Palpation on the buccal region we found a well circumscribed mass with solid consistency which is about $5 \mathrm{~cm}$ in diameter that can be moved from it base. There was no pain on palpation instead of numbness at the right buccal region. Air passage on both nostrils were within normal limit, there was no nasal obstruction. The eyes were within normal limit and the interpuppilary line was in the equal height (Figure 1). Intraoral examination we found palpable mass around vestibular area of the maxillary right premolars that was firm in consistency. There was no other abnormalities in the oral region. The dentition status shows retained root of maxillary right second molar and maxillary left first molar and several missing teeth (Figure 2). We perform ortopantomograph $\mathrm{x}$-ray to find out whether there is bone or sinus involvement. The bone and sinus appearance 
from the OPG were within normal limit, there was no litic or sclerotic lesion of the bone and there was no abnormal appearance on both maxillary sinuses. We found retained root of maxillary right second molar and maxillary left first molar (Figure 3). Then the patient underwent surgery for incisional biopsy before definitive treatment planned. The histopathologic report was confirmed that the diagnosis is a schwannoma, with the appearance of distinctive multiple rounded masses of elongated spindle cells with palisaded nuclei (Antoni A tissue) (Figure 4). For the definitive treatment we performed surgical excision of the schwannoma mass. The excision was performed via intraoral vestibular approach (Figure 5A). After the vestibular incision we perform exploration through the infraorbital area and dissect the mass from surrounding tissues. At the time of exploration, the mass was observed to emanate from the terminal of the infraorbital nerve trunk. From the surgical excision we found solid encapsulated masses with irregular form, size $5 \times 5 \times 2 \mathrm{~cm}$. The mass appears to have a smooth surface with slight lobulated appearance. After the removal of the mass, operation site was closed by suture (Figure 5B) (Figure $5 \mathrm{C})$. One month control after surgery we found no significant sequelle after surgical excision, but we found numbness on the right buccal region that already felt by the patient since the admission. There was no signs of reccurence, the facial appearance appears to be normal. Palpation on the buccal region we found no more other lumps, whether extraorally or intraorally.
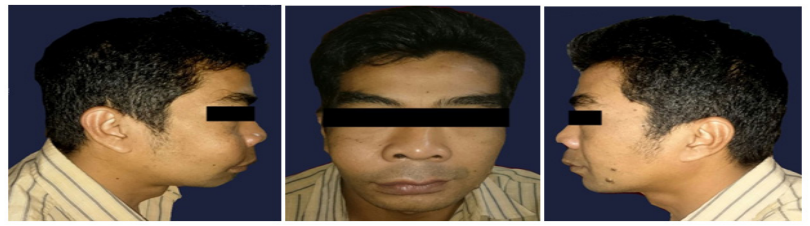

Figure 1 Clinical appearance of the patient.

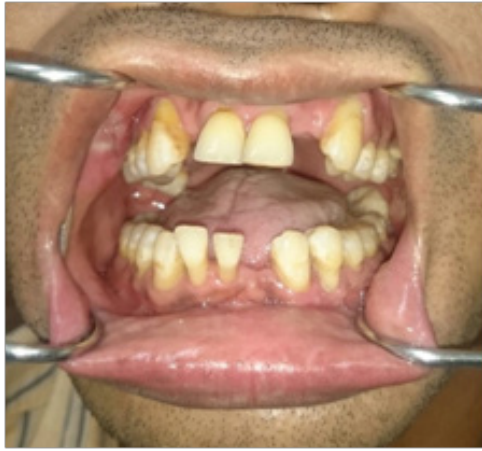

Figure 2 intraoral appearance

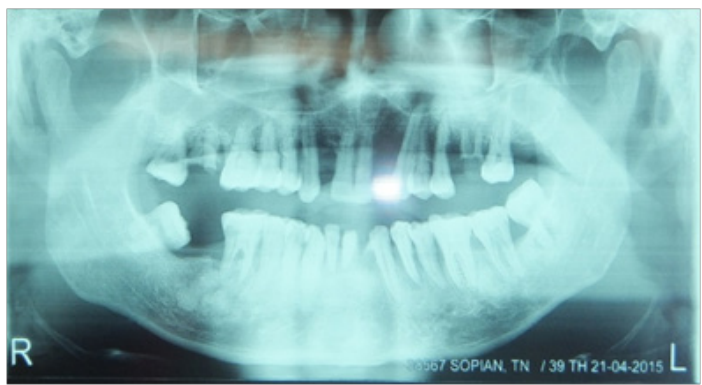

Figure 3 Orthopantomograph $\mathrm{x}$ ray of the patient.

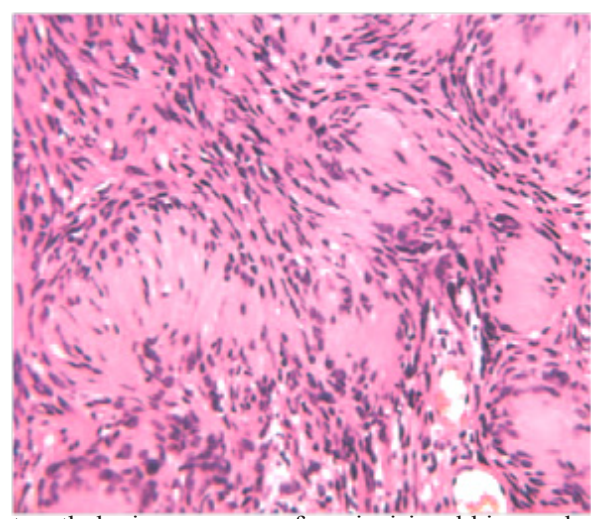

Figure 4 Histopathologic appearance from incisional biopsy showing Anton

A tissue.
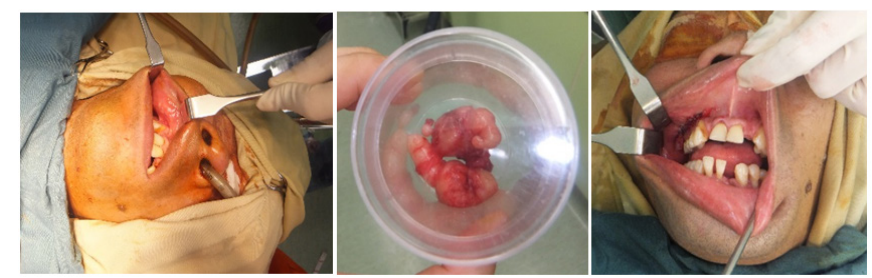

Figure 5(A) Surgical excision was performed via vestibular approach; (B) mass that had been removed; $(\mathrm{C})$ Clinical picture after mass excision.

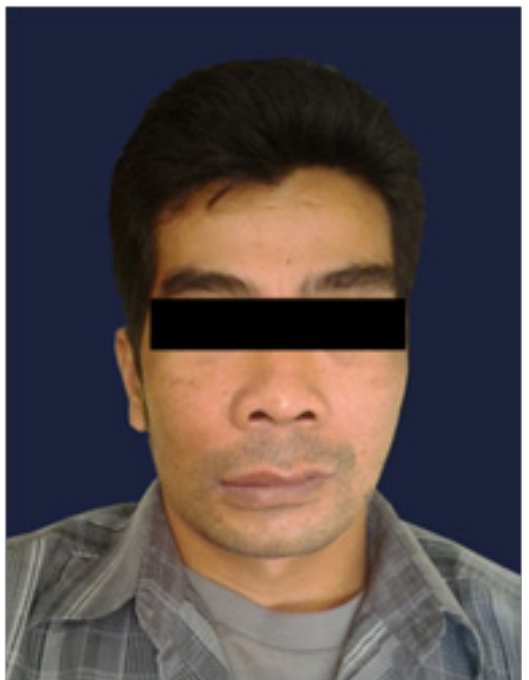

Figure 6 Postoperative clinical picture after 1 month surgery.

\section{Discussion}

Schwannoma arising from the infraorbital nerve trunk is a rare case, lesions in head and neck region account for $25-45 \%$ of extracranial schwannomas. ${ }^{2}$ However, it rarely involves infra-orbital nerve and are uncommon in the maxillary and buccal space accounting for less than $4 \%$. From the history taking and clinical examination show mass that suspected to be a benign tumor. We only perfom simple radiographic imaging which is an orthopantomograph due to based on our history taking and clinical examination we can conclude that 
the tumor is above the bone surface, which is zygoma and no sinus involvement and bone destruction. Treatment of solitary schwannoma is simply by surgical excision and access to the mass determined by its location and structures that involved. In other case report, usually extraoral approach is performed. ${ }^{5,6}$ However, in this case vestibular approach was selected and the main advantages is no post operation scars appears at the facial region.

\section{Conclusion}

Schwannoma of the infraorbital nerve presenting as an upper jaw swelling is a rare entity. Despite its rarity, schwannoma of the infraorbital nerve should be considered in the differential diagnosis of a painless slow growing cheek swelling. The vestibular approach is a safe, effective, and cosmetically acceptable approach for removal of infraorbital nerve trunk schwannoma. An appropriate diagnostic work up includes appropriate imaging and pre-surgical analysis to decide on the best surgical approach.

\section{Acknowledgment}

None.

\section{Conflict of interest}

The author declares that there is no conflict of interest.

\section{References}

1. Marx RE, Stern D. Oral and Maxillofacial pathology: A Rational for diagnosis and treatment. $2^{\text {nd }}$ ed. Quintessence publishing Co, Inc; 2012.

2. Neville BW, Damm DD, Allen CM. Oral and Maxillofacial Pathology. $3^{\text {rd }}$ ed. Saunders Elsevier; 2008.

3. Cawson RA, Odell EW. Cawson's essentialsof oral pathology and oral medicine. $8^{\text {th }}$ ed. Churchill livingstone elsevier; 2008.

4. Sapp JP, Eversole LR, Wysocki GP. Contemporary Oral and Maxillofacial Pathology. $2^{\text {nd }}$ ed. Mosby; 2004.

5. Kumar N. Infra-orbital nerve schwannoma: Report and review. J Nat Sci Biol Med. 2015;6(1):267-271.

6. Garg R, Dhawan A, Gupta N, et al. A rare case of benign isolated schwannoma in the inferior orbit. Indian J Opthalmol.2008;56(6):514-515. 